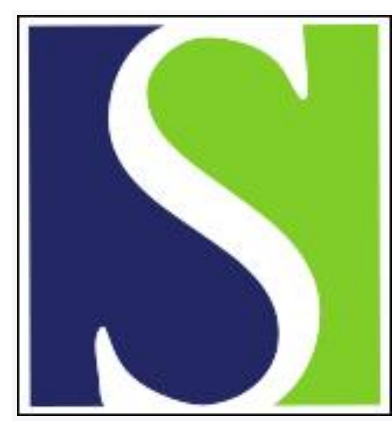

Scand J Work Environ Health 2002;28(3):137-140

https://doi.org/10.5271/sjweh.657

Issue date: Jun 2002

\title{
Can health promotion at the workplace help prevent cancer?
}

by Vainio $H$, Stayner $L$

Affiliation: Unit of Chemoprevention, International Agency for Research on Cancer, Lyon, France.

Refers to the following text of the Journal: 2002;28(3):141-157

Key terms: cancer; editorial; health promotion; prevention; workplace

This article in PubMed: www.ncbi.nlm.nih.gov/pubmed/12109552

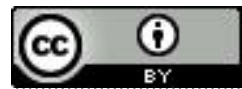




\section{Can health promotion at the workplace help prevent cancer?}

The workplace has become an increasingly important site for disseminating health information and implementing health promotion activities. The creation of "healthy workplaces" must include the prevention of occupational disease and injury, as well as the promotion of positive healthy life-style behavior. Approaching cancer prevention in a comprehensive manner at the workplace is not only scientifically sound, but also sound public health policy. Complementing the reduction of exposures to carcinogens in the workplace with reductions in exposures under the control of individual choice will maximize the potential for occupational cancer prevention, since the many risks of a person are often additive or multiplicative. The most-exciting developments in experimental and epidemiologic research during the last few years are the leads being gained regarding factors that may reduce cancer risk. As we have learned more about such factors as energy balance, physical activity, and specific constituents of the diet and their relationship to human cancer risk, the potential for more general approaches to cancer prevention seems increasingly possible. The concept of creating healthy workplaces through workplace health promotion has been identified as a legitimate area of activity for public health policy as a means of improving the health and well-being of the population at large $(1,2)$. Benefits accrue to businesses, organizations, and individual persons from the enhancement of positive healthy life-style messages in addition to the reinforcement of the principles of good occupational health and safety practices. Commitment to delivering the concept is required from all the key players, who include employers, employees, trade union groups, and health and safety professionals. A healthy workplace model needs to be created that is flexible and adaptable to suit all types of business and, in particular, the needs of the small and medium-size workplaces that predominate in the United States and many European countries.

But how strong is the evidence that health promotion intervention at workplaces really works in disease prevention? Janer and her co-workers (3) have reviewed health promotion trials at workplaces on major cancer risk factors, including tobacco, diet, physical inactivity, obesity, ultraviolet light, and alcohol consumption. The overall evidence indicates a modest positive effect, the clearest for tobacco smoking and positive but small for diet and physical activity.

More than one in four adults in industrialized countries smoke. The health risks due to smoking are important, and the risk is large: approximately $16 \%$ of all cancer deaths in developed countries are attributable to smoking (4). Stopping smoking works remarkably well in reducing cancer risk, as long as it is done before the disease appears (5). Even at middle age (35-69 years), those who stop before serious disease has appeared avoid most of their risk of death from tobacco smoking, and stopping before middle age works even better (6). The worksite provides an ideal setting for smoking cessation programs: an accessible population, an opportunity for participation, and an environment in which coherent messages can be conveyed. Ideally cognitive-behavioral intervention coupled with effective pharmacotherapy may produce up to $40 \%$ quit rates, particularly for those least addicted, most highly motivated, and without psychiatric comorbidity (7). However, the quit rates in worksite trials have been much less than the ideal $40 \%$. The average quit rates in the studies reviewed by Janer and her coworkers was only $6.1 \%$ (range $0.9 \%$ to $12 \%$ ) greater than that of the control group (3). The recidivism rate was found to be $40 \%$ to $80 \% 6$ months after the cessation of the intervention. Although these results are not as large as one might hope for, they appear to have been achieved with very limited efforts that did not include pharmacotherapy. 
Avoidance of weight gain should become one of the mainstays of chronic disease prevention in modern societies. The high prevalence of and increasing trend towards obesity in industrially developed countries can be explained by a life-style characterized by the overconsumption of energy combined with low levels of physical activity. These changes in life-style are a consequence of economic, social, and technological changes, including increased automation and computerization in workplaces and in domestic chores, a reduction in walking and cycling for transport, a decrease in recreational exercising, and a rise in sedentary leisure-time activities such as watching television (8).

Obesity consumed $8 \%$ of the health care budget in the United States and an estimated USD 100 billion in direct and indirect costs in 1995 (8). It is well-known that obesity increases the risk of cardiovascular diseases, but its role in oncogenesis has been less well appreciated. There is strong evidence for a causative role of obesity in cancer of the colon, breast in postmenopausal women, endometrium, and kidney (renal-cell) and adenocarcinoma of the esophagus and gastric cardia (9). Uncertainties remain about the level of weight excess that must be considered a risk factor for cancer.

Weight control can be achieved both through changes in energy intake and through increased physical activity (9). Promoting physical activity in combination with dietary changes seems particularly effective in the prevention of weight gain, while results are modest when physical activity only, without any dietary restriction, is employed to achieve weight reduction. About half of the work-aged adults in industrialized countries are physically inactive or underactive, and at least half of the youth are not vigorously active on a regular basis. Physical inactivity prevalence is thus about twice that of smoking in industrialized countries, and both risk factors are associated with substantial morbidity and mortality. The major benefits of physical activity, above and beyond weight control, are well known, including a decrease in all-cause mortality rates and a decrease in the risk of cardiovascular diseases and diabetes. There is also growing evidence that regular physical activity, during work and leisure time, reduces cancer risk, and this association appears to be independent of that of cancer risk with body weight. Thus it has been estimated that 30 to 60 minutes of physical activity daily may reduce the risk of colon, breast, endometrial, and prostate cancer by $20 \%$ to $40 \%$ (9).

The results from the intervention studies on physical activity and weight loss that have been reviewed by Janer and her co-workers (3) are more mixed than the results for smoking cessation programs. Only 7 of 13 studies of physical activity programs showed significant improvements. It is notable that the success rate appears to be much lower among programs that offer facilities, time, and space for the activities than among those that merely offer educational information. Studies of weight-loss programs demonstrated small-to-moderate amounts of loss (range +0.25 to -3.5 kilograms). However, the persistence of weight loss was only assessed in one study. Although this study did not report any reduction in effect after 1 year, there are clearly inadequate data with which to evaluate the long-term effectiveness of these programs. An ideal outcome of weight control intervention would be a return of body weight to the normal range with no weight gain thereafter. This is an unrealistic goal for most conditions - and demonstrates the great "addictive strength" of being overweight. It seems more difficult to lose weight, permanently, than to stop smoking.

Overall, there is some evidence of a modest effect of health promotion programs at work, such as smoking cessation, increase in physical activity, weight control, and intake of fruits and vegetables (3). Questions remain about how persistent the effects are, and there is a definite need for more studies that measure both changes in behavior and health outcomes over a long follow-up period. It does appear that there is a real risk of recidivism by smokers and for other life-style changes and that effective programs need to be continuous rather than short-term. It is also important to note that these programs should not be conducted in place of or at the expense of other programs designed to reduce occupational health and safety hazards found in the workplace. Too often, in the workforce, these efforts may be competing for limited resources. In fact, health promotion programs and programs aimed at reducing workplace 
hazards may often be complimentary. For example, smoking (active or passive) may interact with workplace exposures to induce lung cancer, and alcohol consumption may be a contributor to workplace accidents (10).

Cancer prevention research must coordinate the diverse disciplines of public health with those of behavior and social sciences. This integration of disciplines is essential to the development of the studies required to understand both individual and community responses to the changes in diet, smoking, physical activity, and other personal and cultural practices that are necessary to achieve the goals of cancer prevention. But the limited impact of the programs seeking to motivate behavior change at the individual level is noteworthy, and it is illustrated by the typically modest results, if any, of efforts to get people to lose weight or lower their intake of fats. Swimming against the prevailing tide is difficult for the individual (particularly for the socially disadvantaged!), and changing the direction of the tide is difficult for policy makers and opinion leaders.

\section{References}

1. Biener L, Glanz K, McLerran D, Sorensen G, Thompson B, Basen-Enguist K, Linnan L, Varnes J. Impact of the Working Well Trial on the worksite smoking and nutrition environment. Health Educ Behav 1999;26:478-94.

2. Steenhuis IH, van Assena P, Glanz K. Strengthening environmental and educational nutrition programmes in worksite cafeterias and supermarkets in the Netherlands. Health Promot Int 2001;16:21-33.

3. Janer G, Sala M, Kogevinas M. Health promotion trials at worksites and risk factors for cancer. Scand J Work Environ Health 2002;28(3):141-57.

4. Peto R, Lopez A, Boreham J, Heath C, Thun M. Mortality from tobacco in developed countries 1950-2000. Oxford: Oxford University Press, 1994.

5. Vainio H, Weiderpass E, Kleihues P. Smoking cessation in cancer prevention. Toxicology 2001;166:47-52.

6. Peto R, Darby S, Deo H, Silcocks P, Whitley E, Doll R. Smoking, smoking cessation, and lung cancer in the UK since 1950: combination of national statistics with two case-control studies. Br Med J 2000;321:323-9.

7. Westmaas JL, Nath V, Brandon TH. Contemporary smoking cessation. JMCC 2000;7:56-62.

8. Wolf AM, Colditz GA. Current estimates of the economic costs of obesity in the United States. Obes Res 1998;6:97106.

9. International Agency for Research on Cancer (IARC). Weight control and physical activity. Lyon: IARC Press, 2002:1315. IARC handbooks of cancer prevention, vol 6.

10. Cohen A, Murphy L. Indicators of health promotion behaviors in the workplace. In: Kar S, editor. Health promotion indicators and actions. New York (NY): Springer Publishing Co, 1998:chapter 12.

Harri Vainio, MD

Unit of Chemoprevention

International Agency for Research

on Cancer

Lyon, France and

Leslie Stayner, PhD
Visiting Scientist
International Agency for Research
on Cancer
Lyon, France

